\title{
Diskursive Konstruktionen von Europa
}

\author{
Eine Integration von Öffentlichkeits- und Identitätsforschung
}

\section{Christiane Eilders / Dennis Lichtenstein}

Der Beitrag knüpft an die mangelnde Unterstützung der EU durch die Bevölkerungen der Mitgliedsstaaten an und erweitert die in der kommunikationswissenschaftlichen Forschung häufig auf die europäische Öffentlichkeit eingeschränkte Ursachenforschung um die Dimension der europäischen Identität. Im Mittelpunkt des Beitrags steht eine theoretische Neukonzeption der EU-bezogenen Identitätsforschung durch eine öffentlichkeitstheoretische Fundierung. Die kollektive Dimension von Identität steht dabei im Mittelpunkt. Die Neukonzeption geht davon aus, dass die kollektive Identität einer Gesellschaft in den nationalen öffentlichen Diskursen unter Einbeziebung verschiedener Sprecher konstruiert wird. Sie lässt sich wie auch die öffentliche Meinung als gesellschaftliches Diskurs-Resultat begreifen, das über den Mediendiskurs einfach wabrnebmbar ist und auch politische Entscheidungen anleiten kann. Die europäische Identität umfasst in diesem Konzept zum einen das Zugehörigkeitsgefühl zur EU, also das Selbstverständnis einer Gesellschaft als Teil der EU, zum anderen die inhaltliche und das Zugebörigkeitsgefübl begründende Ausdeutung der EU als spezifische Art von Gemeinschaft, z. B. als wirtschaftliche, politische und kulturelle Gemeinschaft oder als geografische Einheit.

Schlagwörter: Europäische Identität, kollektive Identität, Europäische Öffentlichkeit, Diskurs, Framing

\section{Einleitung}

Das politische Zusammenwachsen Europas hat während der letzten Jahrzehnte erheblich an Dynamik gewonnen. Dies betrifft sowohl die Verlagerung wesentlicher Entscheidungskompetenzen von der nationalen auf die supranationale Ebene als auch die Erweiterung der EU um eine Reihe neuer Mitgliedsstaaten. Während Eliten in Politik und Wirtschaft die Intensivierung der Zusammenarbeit in der EU zumeist befürworten und aktiv vorantreiben, zeigen sich in breiten Bevölkerungsschichten vor allem Desinteresse, Skepsis und Widerstand. Diese ablehnende Haltung wird besonders deutlich in der regelmäßig schwachen Beteiligung der EU-Bürger an den Wahlen zum europäischen Parlament sowie zuletzt in den gescheiterten Referenden zum europäischen Verfassungsvertrag 2005 in Frankreich und den Niederlanden bzw. 2008 in der ersten Abstimmung zum Vertrag von Lissabon in Irland. Diese Beispiele müssen als Symptome für ein schwaches Zugehörigkeitsgefühl der EU-Bürger gewertet werden und weisen auf eine Spaltung zwischen Eliten und breiter Bevölkerung hin. Auch nach 50 Jahren Integrationspolitik identifizieren sich die Bevölkerungen der Mitgliedsstaaten noch immer nicht eindeutig mit der EU.

Für die Legitimität des politischen Verbandes EU genügt es nicht, wenn sich lediglich die Eliten als EU-Bürger fühlen. Auch bei der breiten Bevölkerung muss sich als unverzichtbare Legitimitätsressource eine europäische Identität ${ }^{1}$ herausbilden. Eine soziale

1 Der Begriff „europäische Identität“ und der Begriff der „europäischen Öffentlichkeit“ ist hier und im Folgenden in einem engen Verständnis bezogen auf die EU und nicht auf den europäischen Kontinent zu begreifen. 
Identität als EU-Europäer, die sich zunächst auf das individuelle Zugehörigkeitsgefühl zur EU bezieht, stärkt die Gemeinschaft bereits deshalb, weil sich auf Grundlage dieser Selbstwahrnehmung eine Solidarität mit anderen EU-Bürgern entwickeln kann. Wie in jeder Demokratie ist nämlich mit einer gemeinsamen sozialen Identität nicht nur das Bewusstsein verbunden, sich auf gleiche staatsbürgerliche Rechte berufen zu können, sondern zugleich die Bereitschaft, innerhalb der EU Pflichten zu erfüllen, Opfer zu bringen und Mehrheitsentscheidungen zu akzeptieren. Je stärker das Zugehörigkeitsgefühl zur EU ausgeprägt ist, desto wahrscheinlicher ist es, dass die EU-Europäer sich gegenüber den EU-Institutionen loyal verhalten, am öffentlichen Diskurs teilnehmen und damit zur Stabilität der europäischen Gemeinschaft beitragen.

Das Identitätsdefizit innerhalb der EU wird derzeit flankiert von einem Demokratieund einem Öffentlichkeitsdefizit. Beide verweisen auf eine mangelnde Rückbindung der politischen Entscheidungsprozesse an die Bevölkerungen der Mitgliedsstaaten und stellen insofern ebenfalls die Legitimität der Gemeinschaft in Frage. Das Identitätsdefizit einerseits und das Demokratie- und das Öffentlichkeitsdefizit andererseits beeinflussen sich gegenseitig: Während mangelnde Partizipationsmöglichkeiten in der EU sowie die schwach ausgeprägte europäische Öffentlichkeit einem Identitätsgefühl als EU-Bürger entgegenwirken (Habermas 2004), reduziert die fehlende europäische Identität den Willen zur Partizipation und zum transnationalen öffentlichen Diskurs (Kielmansegg 1996).

Der vorliegende Beitrag führt die Konzepte Öffentlichkeit und Identität zusammen und entwickelt unter Rückgriff auf öffentlichkeitstheoretische Überlegungen eine konzeptionelle Erweiterung der Identitätsforschung. Dabei wird die Problematik der europäischen Identität anhand einschlägiger Ansätze und empirischer Befunde illustriert, bevor die möglichen Schnittstellen zwischen den zentralen Forschungstraditionen zur europäischen Öffentlichkeit und zur europäischen Identität diskutiert werden. Die Ansätze zur europäischen Öffentlichkeit (Abschnitt 2) und zur europäischen Identität (Abschnitt 3) sind bislang trotz bestehender Anschlussmöglichkeiten weitgehend unverbunden geblieben. Der Beitrag integriert die Forschungsstränge (Abschnitt 4), indem er das Konzept europäischer Identität öffentlichkeitstheoretisch fundiert und für die Makroperspektive auf Gesellschaft und Politik zugänglich macht. Damit richtet sich der Blick auf die kollektive Identität der einzelnen Mitgliedsstaaten. Mit der Neukonzeption ist ein erweiterter methodischer Zugriff auf die europäische Identität verbunden, der die gesellschaftlichen Identitätsdiskurse in den Mittelpunkt rückt. In einem abschließenden Fazit wird das theoretische und empirische Potenzial der hier entwickelten Neukonzeption diskutiert (Abschnitt 5).

\section{Europäische Öffentlichkeit: Ansätze und Befunde}

Mit der Kompetenzerweiterung der EU im Zuge der Maastrichter Verträge, auf deren Grundlage sich die Wirtschaftsgemeinschaft EG zu einem starken politischen Regime gewandelt hat, hat sich das Interesse der Forschung zunehmend auf die demokratietheoretischen Anforderungen an die EU gerichtet. Die Sozialwissenschaften stehen vor der Herausforderung, ihren bisher für nationale Gesellschaften konzipierten Referenzrahmen auf die europäische Dimension zu erweitern und ihre auf nationaler Ebene bewährten Konzepte zu modifizieren (Neidhardt 2006). Ein Schwerpunkt der Forschungsaktivitäten liegt derzeit auf den Entstehungsbedingungen einer europäischen Öffentlichkeit.

Während die nationalstaatlich organisierten Demokratien über die nationalen Mediendiskurse Öffentlichkeit herstellen, hinkt die Entwicklung einer europäischen Öffentlichkeit der transnationalen Entgrenzung anderer gesellschaftlicher Systeme hinterher 
(Gerhards 2000). Die auf europäischer Ebene vergleichsweise schwach entwickelten Strukturen einer politischen Öffentlichkeit sind damit demokratietheoretisch höchst problematisch. Da eine mit der nationalen Öffentlichkeit vergleichbare paneuropäische Variante aufgrund der Sprachenvielfalt (Kielmansegg 1996) und national verwurzelter Mediensysteme allenfalls als Elitenöffentlichkeit denkbar ist (z. B. Eilders/Voltmer 2004), wird die Verschränkung des politischen Bereichs mit dem Geltungsbereich der Öffentlichkeit auf der supranationalen Ebene aufgelöst (Imhof 2003).

In Reaktion auf das Fehlen einer gesamteuropäischen Öffentlichkeit konzentriert sich die Forschung auf die Europäisierung der nationalen Öffentlichkeitsarenen. Aber auch hier muss die Forschung mit einem Elite-Bias umgehen: Europabezüge finden sich insgesamt weniger in den von der breiten Bevölkerung genutzten Medien als in solchen, die sich an die gesellschaftlichen Eliten richten. Da sich die Öffentlichkeitsforschung bislang stark auf die überregionale Presse konzentriert, gerät damit vor allem der Elitendiskurs in den Blick.

In den Untersuchungen nationaler Medien lässt sich eine vertikale und eine horizontale Europäisierungsdimension unterscheiden (Koopmans 2004). In vertikaler Perspektive wird die Europäisierung von Öffentlichkeit als Zunahme von EU-Bezügen in den nationalen Arenen begriffen. Gemessen wird dabei die Aufmerksamkeit der Medien für europapolitische Themen und Akteure im Zeitverlauf und/oder im Verhältnis zur gesamten Politikberichterstattung (z. B. Eilders/Voltmer 2003, Gerhards 2000, Trenz 2004). Diese Untersuchungen fragen nach der Transparenz politischer Entscheidungsprozesse auf EU-Ebene und nach der Sichtbarkeit des Einflusses europäischen Regierens auf die nationale Ebene. In horizontaler Perspektive werden demgegenüber die Aufmerksamkeit für andere europäische Staaten sowie Vernetzungen zwischen den nationalen Mediendiskursen untersucht. So analysieren Eder und Kantner (2000: 315), ob in den Medien „zur gleichen Zeit die gleichen Themen unter den gleichen Relevanzkriterien" verhandelt werden.

In Bezug auf die vertikale Dimension finden Trenz (2004), Engelmann (2009) und Sievert (1998) sowie Wessler und seine Kollegen (2008) einen vergleichsweise starken Europäisierungsgrad. Demgegenüber beobachten Gerhards (2000) sowie Eilders und Voltmer (2003) zwar eine leicht zunehmende Europäisierungstendenz seit Mitte der 1990 er Jahre, dies aber auf sehr niedrigem Niveau. Gering fällt die Berichterstattung über die EU vor allem dann aus, wenn keine EU-Schlüsselereignisse stattfinden, während z. B. Gipfeltreffen einen moderaten Anstieg der Medienaufmerksamkeit bewirken (Peter/de Vreese 2004). Dabei vollziehen sich die vertikalen Europäisierungsprozesse politikfeldspezifisch und in Abhängigkeit von den jeweiligen Entscheidungskompetenzen der EU innerhalb der Politikfelder (Koopmans 2004).

In der horizontalen Dimension zeigt sich, dass über das EU-Ausland nicht intensiver berichtet wird als über nicht-europäische Länder (Kalantzi 2004). Aufmerksamkeit finden in der EU vor allem die wirtschaftlich und politisch starken Mitgliedsländer und deren Repräsentanten, wobei auch die jeweilige geografische und kulturelle Nähe zu den anderen Ländern eine Rolle spielt (ebd.). In Bezug auf die Entwicklung über die Zeit zeigt sich keine wesentliche Zunahme an Querbezügen zwischen den Mitgliedsstaaten (Wessler et al. 2008). Lediglich Engelmann (2009) diagnostiziert jedenfalls für die deutsche Medienlandschaft eine leichte Zunahme in der horizontalen Europäisierung.

Hinsichtlich der spezifischen Perspektiven, unter denen gemeinsame Themen diskutiert werden, ergibt sich ein uneinheitliches Bild. So zeigen Framing-Analysen zum Kosovo-Konflikt 1999 (Grundmann et al. 2000), zum Skandal um die Santer-Kommission (Trenz 2000) und zum Fall Schulz-Berlusconi (Downey/König 2006) im Länder- 
vergleich, dass überwiegend nationale Perspektiven auf die Themen angelegt werden. Transnational übereinstimmende Frames belegen dagegen z. B. die Untersuchungen zum „Fall Haider“ (Risse/van de Steeg 2003), zur Osterweiterung (van de Steeg 2003) sowie zu den Debatten um die Themen Schengen II, Korruptions- und BSE-Skandal (Eder/Kantner 2000).

Die Gesamtheit der Studien macht deutlich, dass die z. B. von Grimm (1995) vertretene Auffassung, eine kontrollierende, integrierende und zur Meinungsbildung befähigende Öffentlichkeit könne sich im transnationalen europäischen Raum auch längerfristig nicht herausbilden, in dieser Allgemeinheit revidiert werden muss. Nationale und transnationale Öffentlichkeiten müssen als ineinander verschachtelte politische Kommunikationsräume gedacht werden (Neidhardt 2006). Obwohl innerhalb der EU transnationale Kommunikationszusammenhänge bestehen, bleiben die kommunikativen Verdichtungen innerhalb der nationalen Öffentlichkeiten deutlich stärker, woraus sich insgesamt der Befund einer national segmentierten europäischen Öffentlichkeit ergibt (Hepp/Wessler 2009). Vernetzungen zwischen den nationalen Arenen finden sporadisch und vor allem durch Konfliktereignisse stimuliert statt. Konfliktkommunikation gilt damit als wirksamer Integrationsmechanismus (Berkel 2006) und intensiviert sowohl die vertikale als auch die horizontale Europäisierung. Entsprechend lässt sich mit Kantner (2004) aus den zitierten Studien ein Öffentlichkeitsverständnis ableiten, das europäische Öffentlichkeit als variables Netzwerk von themen- und ereigniszentrierten Teilöffentlichkeiten beschreibt. Trotz der Hinweise auf diese sporadischen Kommunikationsverdichtungen werden stets Defizite aufgezeigt. Nie wird behauptet, „Europa verfüge bereits über eine ausreichend breite, tragfähige politische Öffentlichkeit" (Saurwein et al. 2006: 5). Es lässt sich also insgesamt bezweifeln, dass die vorhandene europäische Öffentlichkeit hinreichend entwickelt ist, um einerseits die europäischen Entscheidungsprozesse transparent $\mathrm{zu}$ machen und zu kontrollieren und andererseits die europäischen Gesellschaften $\mathrm{zu}$ einer Kommunikationsgemeinschaft $\mathrm{zu}$ integrieren, in der sich die Entwicklung einer europäischen Identität beschleunigt.

Identitätsfragen spielen in der Öffentlichkeitsforschung nur am Rande eine Rolle (Brüggemann et al. 2009). Aussagen über das Ausmaß europäischer Identität erlauben nur wenige quantitative Medieninhaltsanalysen, die explizite „Wir-Bezüge“ und Nennungen von Kollektivnamen in Medienbeiträgen als Artikulationen einer europäischen Identität werten (Brantner 2009, Lucht/Tréfas 2006, Wessler et al. 2008). Insgesamt zeigen diese Studien eine Stagnation auf niedrigem Niveau. Dabei hängt die Häufigkeit der "Wir-Bezüge“ vom thematischen Kontext ab; so verweisen Fallstudien innerhalb der Langzeitstudie von Wessler und Kollegen (2008) auf einen leicht zunehmenden Anteil europäischer Identitätsartikulationen. Die in diesen Studien unternommene empirische Erfassung der Identitätsartikulationen in Mediendiskursen stellt innerhalb der Öffentlichkeitsforschung bisher die Ausnahme dar. Auch die inhaltliche Ausdeutung der EU als soziale Gemeinschaft gerät nur selten in den Blick von Inhaltsanalysen, ${ }^{2}$ und zwar obwohl in theoretischen Arbeiten immer wieder darauf hingewiesen wird, dass Gemeinschaftsbildung im Zuge öffentlicher Kommunikation stattfindet (Imhof 2003).

Wie zu zeigen sein wird, steht der Prozess gesellschaftlicher Gemeinschaftsbildung im Zuge öffentlicher Kommunikation allerdings auch in der Identitätsforschung nicht im Mittelpunkt. In der Tradition der politikwissenschaftlichen Meinungs- und Einstellungsforschung wird eher nach individuellen Zugehörigkeitsgefühlen zur EU gefragt,

2 Eine Ausnahme ist die bereits angesprochene Untersuchung der Haider-Debatte von Risse und van de Steeg (2003). 
wie sie über Umfragen ermittelt werden. Weiterführend ist die Forschung zu sozialen Bewegungen, die kollektiven Identitäten und den zur Partizipation motivierenden Frames eine zentrale Bedeutung zuweist (Gamson et al. 1982, Snow et al. 1986, Snow/ Benford 1992, Gerhards/Rucht 1992). Empirische Studien des Mediendiskurses bleiben dabei meist auf bewegungs-relevante Themen beschränkt und beziehen sich damit auf soziokulturelle Kontexte unterhalb der gesamtgesellschaftlichen Makroebene. Während die Öffentlichkeitsforschung also zwar mit Makrokonzepten arbeitet, dabei aber Identitätsfragen weitgehend ausklammert, befasst sich die Identitätsforschung entweder mit der individuellen Zugehörigkeit zur EU oder mit der kollektiven Zugehörigkeit zu soziokulturellen Einheiten, nicht aber zum supranationalen Gebilde der EU.

\section{Europäische Identität: Ansätze und Befunde}

Neben der Entwicklung einer gemeinsamen Öffentlichkeit ist auch das Zugehörigkeitsgefühl der nationalen Bevölkerungen von der transnationalen Entgrenzung betroffen. Während der wissenschaftliche Diskurs zur europäischen Öffentlichkeit bereits umfangreiche Forschungsaktivitäten verzeichnet, rückt das Thema der europäischen Identität erst seit ein paar Jahren stärker in den Fokus (z. B. in Herrmann et al. 2004). Relevant ist die Frage nach einer europäischen Identität spätestens seit der Erweiterung der Wirtschaftsgemeinschaft EG zu dem supranationalen Regierungssystem EU. Zwar existiert die Idee eines europäischen Zugehörigkeitsgefühls bereits seit der Antike, sie bezog sich bis Mitte des 20. Jahrhunderts aber vor allem auf den europäischen Kontinent und dessen Bevölkerung (z. B. Schmale 2008). In demokratietheoretischer Perspektive muss sich die Frage nach einer europäischen Identität nun aber konkreter auf die EU als politisches System richten.

Mit dem Konzept der europäischen Identität befasst sich vor allem die politikwissenschaftliche Meinungs- und Einstellungsforschung. In neueren Arbeiten wird dabei auf sozialpsychologische Ansätze zurückgegriffen (z. B. Breakwell 2004, Castano 2004), die soziale Identität als Zugehörigkeitsgefühl eines Individuums zu einer Gruppe bestimmen und dieses als Teil der Selbstdefinition eines Individuums betrachten (Brewer 1991). In einer konstruktivistischen Perspektive wird hier davon ausgegangen, dass personale Identitäten nicht statisch sind, sondern je nach Kontext unterschiedliche Gruppenzugehörigkeiten an Relevanz gewinnen (Brewer 2009). In Bezug auf politische Gemeinschaften begründet die empfundene Zugehörigkeit die Loyalität des Individuums gegenüber den Institutionen und Regeln der Gemeinschaft sowie seine Bereitschaft zur politischen Partizipation. Für die Stabilität der EU ist es daher von großer Bedeutung, wie stark sich ein Individuum der EU zugehörig fühlt. Entsprechende Indikatoren werden regelmäßig in den Umfragen des „Eurobarometers“ erfasst, das die Europäische Kommission zweimal jährlich in Auftrag gibt. Das Eurobarometer stellt damit die umfangreichste Datenquelle zu den identitätsrelevanten Einstellungen in den EU-Mitgliedsländern dar.

Typische Fragekomplexe richten sich auf die Zustimmung der Bürger zur EU-Mitgliedschaft ihres Landes, auf die individuell empfundene europäische und nationale Identität sowie auf das Wissen und das Informationsbedürfnis zur EU. Die Befunde zeigen, dass die Unterstützung für die EU nach einem Anstieg zwischen 1980 und 1990 in der folgenden Zeit wieder stark zurückgegangen ist. Eichenberg und Dalton (2007) bezeichnen dieses Phänomen als „Post-Maastricht-Blues“. Nach den letzten Ergebnissen, die sich auf die Befragung im Herbst 2009 beziehen, liegt die Befürwortung der 
nationalen Mitgliedschaft zur EU im Durchschnitt der EU-Staaten ${ }^{3}$ nur bei 53 Prozent (Eurobarometer 72). Auch das Zugehörigkeitsgefühl zur EU ist Gegenstand des Eurobarometers und wird über verschiedene Fragen erschlossen. Im Herbst 2007 gaben 49 Prozent der befragten EU-Bürger an, sich der EU im Vergleich zu ihrem Land und ihrer Stadt bzw. Dorf „sehr“ bis „ziemlich“ verbunden zu fühlen (Eurobarometer 68). Auf die Frage, wie häufig sie sich als Europäer fühlen, antworteten im Herbst 2005 17 Prozent der EU-Bürger mit „oft“ und 38 Prozent mit „manchmal“ (Eurobarometer 64). Dabei zeigen sich beträchtliche Länderunterschiede. Am häufigsten gab die Bevölkerung in Luxemburg an, sich oft europäisch zu fühlen (38\%), am seltensten die Bevölkerung in Großbritannien (8 \%). Differenzen im Ausmaß des Zugehörigkeitsgefühls zwischen den Nationen zeigen auch die qualitativen Interviews von Díez Medrano (2003), wobei sowohl diese Daten als auch jene des Eurobarometers durchweg darauf hinweisen, dass sich gesellschaftliche Eliten stärker mit der EU verbunden fühlen als die breite Bevölkerung.

Die Defizite in Bezug auf die europäische Identität sind keineswegs der Konkurrenz mit der nationalen Identität geschuldet. Es handelt sich nicht um ein Nullsummenspiel zwischen den Identitäten (z. B. Marcussen et al. 1999). Vielmehr fühlten sich im Herbst 200555 Prozent der Befragten sowohl als Europäer als auch als Bürger ihres Landes (Eurobarometer 64), wobei die EU vor allem als sekundäre Identität neben der nationalen angegeben wurde, sodass die Tendenz lautet: „Country first, but Europe too“ (Risse 2004: 249). Solange die Selbstständigkeit der eigenen Nation nicht als stark beeinträchtigt wahrgenommen wird (vgl. etwa de Vreese/Boomgaarden 2005), können Individuen also mehrere Gruppenzugehörigkeiten in ihr Selbstkonzept aufnehmen, ohne dass es dabei zu Unvereinbarkeiten kommt. Den hybriden Charakter von Identitäten verdeutlicht Risse (2004) anhand des Bildes vom Marmorkuchen, in dem jede Scheibe anders aussieht: Je nach Betonung einzelner Bezugsgrößen - wie etwa der EU, der Nation oder der Region - entstehen unterschiedliche Mischungen von Identität (vgl. auch Breakwell 2004). Die Identität eines Individuums ist demnach immer nur als bestimmtes Verhältnis von verschiedenen Zugehörigkeiten zu unterschiedlichen Gruppen zu beschreiben.

Obgleich in den Eurobarometer-Umfragen die Länderunterschiede und damit größere Einheiten im Vordergrund stehen, wird die Zugehörigkeit zu einer bestimmten Gruppe oder Gemeinschaft meist nur auf der Mikroebene, also bezogen auf individuelle Einstellungen, untersucht. Die kollektive Identität einer Gesellschaft gerät lediglich als Aggregat der Mikro-Daten in den Blick. Dem Verständnis kollektiver Identität als „shared definition of a group that derives from members' common interests, experiences, and solidarity" (Taylor/Whittier 1992: 105, Hervorh. d. Verf.) wird diese Art der Meinungs- und Einstellungsforschung damit nicht gerecht. Sie fokussiert auf die Relevanz der EU für die individuellen Zugehörigkeitsgefühle. Vernachlässigt werden dabei die Konstruktionsmechanismen auf kollektiver Ebene, die eine soziale Gruppe über die Vermittlung von Kontinuität und Kohärenz (Morley/Robins 2002) etablieren und damit erst die Integration von Individuen innerhalb dieser Gruppe ermöglichen. Für die Untersuchung der europäischen Identität in verschiedenen EU-Ländern ist somit nach den Konstruktionsprozessen kollektiver Identitäten zu fragen, in denen die Differenz zu

3 Die Varianz zwischen den Ländern ist hier groß: Ein „europawarmes“ Land ist beispielsweise Luxemburg (79\%), ein „europakaltes“ Lettland (25\%).

4 Seit 2005 wurde dieser Aspekt nicht mehr erhoben. 
anderen Gruppen und die Prinzipien der In- und Exklusion ${ }^{5}$ (Eisenstadt/Giesen 1995) bestimmt werden. Hier sind Anleihen bei der Forschung zu sozialen Bewegungen weiterführend, selbst wenn dort die Konstruktion europäischer Identität eher am Rande behandelt wird.

Soziale Bewegungen können mit einem normativen Projekt nur in dem Maße erfolgreich sein, in dem es ihnen gelingt, ein kollektives „Wir“ mit diesem Projekt zu verbinden (Eder 2000). Das in der Bewegungsforschung entwickelte Framing-Konzept (z. B. Gamson 1992, Snow/Benford 1988) ist für die hiermit angesprochene Konstruktion kollektiver Identität besonders einschlägig. Die Konstruktion kollektiver Identität wird oberhalb der Individualebene in den Diskursen bestimmter soziokultureller Kontexte angesiedelt (Gamson/Meyer 1996) und als Framing beschrieben (Snow/Benford 1992). Über Framing-Analysen von Medieninhalten können diese identitäts-konstituierenden Diskurse vergleichsweise einfach empirisch erfasst werden. Die Tradition unterscheidet drei Bestandteile von Framing: Neben dem diagnostic- und prognosticFraming kann als motivational-Framing die Identitätskonstruktion für den Mobilisierungserfolg zentral werden (Eilders/Lüter 2000: 417). Indem die Bewegungsforschung grenzüberschreitende Gruppen mit gemeinsamen Themeninteressen und Zielen aus einer Identitäts-Perspektive betrachtet und die entsprechenden Kommunikationszusammenhänge als Mechanismen für die Konstruktion kollektiver (Bewegungs-)Identitäten über Identitäts-Framing fasst (Haunss 2004), verbindet sie die Öffentlichkeits- und die Identitätsperspektive. Diese Integration betrifft auch die unterschiedlichen Analyseebenen dieser beiden Forschungstraditionen: Über Anleihen bei der in der Bewegungsforschung verwendeten Konzeptionalisierung des Begriffs „kollektive Identität“ lässt sich die Mikroorientierung der Meinungs- und Einstellungsforschung überwinden und die Kluft zu den Makroansätzen der Forschung zur europäischen Öffentlichkeit verringern.

Da die Bewegungsforschung sich vor allem mit der Identitätskonstruktion soziokultureller Milieus, nicht aber mit der Konstruktion einer themenübergreifenden europäischen Identität befasst, finden sich in dieser Forschungstradition kaum konkrete Hinweise auf mögliche Einflussfaktoren auf die Konstruktion europäischer Identität. Als kollektive Identität ist eine europäische Identität als das Zusammengehörigkeitsgefühl einander nicht persönlich bekannter Individuen innerhalb einer „vorgestellten Gemeinschaft“ (Anderson 2005) zu verstehen. Kollektive Identitäten etablieren sich in ihrer Differenz zu anderen Gruppen (Hall 1994) und ihre Konstruktion wird maßgeblich von Wahrnehmungen geleitet (Wimmer 2003). Mit der Wahrnehmung von Gruppen beschäftigt sich die Sozialpsychologie. Einschlägige Ansätze können damit zur Erklärung der unterschiedlich ausgeprägten europäischen Identität in den EU-Mitgliedsländern beitragen. Nach der Optimal Distinctiveness-Theorie wenden sich Individuen bevorzugt Gruppen zu, die groß genug sind, um ein Sicherheitsgefühl zu vermitteln, und sich gleichzeitig unverwechselbar von bedeutenden anderen Gruppen unterscheiden (Brewer 1991). Demnach müsste eine europäische Identität vor allem dann stark ausgeprägt sein, wenn die EU leicht als kohärente und nach außen eindeutig abgrenzbare Gemeinschaft wahrnehmbar ist.

Campbell (1958) unterscheidet vier relevante Faktoren, die er aus der Gestalttheorie ableitet: Die Wahrnehmung einer Ähnlichkeit der Mitglieder, eines gemeinsamen Schicksals im Sinne einer geteilten Vergangenheit oder Zukunft, klarer Gruppengrenzen und die situative Bedeutsamkeit der Gruppe. Diese Variablen fasst er unter dem Begriff

5 In- und Exklusionen werden über Codes geregelt. Eisenstadt und Giesen (1995) unterscheiden zwischen stabilen Codes wie Ethnie oder Geschlecht, kulturellen Werten, Normen und Traditionen sowie voluntaristischen Bekenntnissen zu gemeinsamen Zielen und Idealen. 
„Entitativity“ zusammen. Die Entitativity bezeichnet damit das Ausmaß, in dem eine Gruppe als real existierend wahrgenommen wird. Wenn die Zugehörigkeit zu einer $\mathrm{Ka}$ tegorie im Alltagsleben relevant und direkt erfahrbar ist, so ist die Entitativity besonders hoch ausgeprägt. In diesem Fall dürfte die Gruppenzugehörigkeit stark empfunden werden. Weil Gruppen mit hoher Entitativity als handlungsfähig und stabil gelten, Schutz bieten und Unsicherheiten reduzieren, wirken sie auf die Individuen attraktiv (Castano et al. 2003). Insbesondere für international vergleichende empirische Untersuchungen bietet das Modell der Entitativity Anschlussmöglichkeiten, weil es bezogen auf Nationalstaaten Determinanten aufzeigt, die kollektive Identitäten maßgeblich beeinflussen können.

Für die EU kann keine ausgeprägte Entitativity vermutet werden. Die europäische Kategorie erweist sich hinsichtlich ihrer konkreten Inhalte als diffus: Sie besitzt keine klare soziale Bedeutung, wenige Symbole, eine kurze Geschichte ohne eindeutig zu benennendes historisches Erbe sowie unklare Grenzen und ist in diesem Sinne eine „,unfinished' category” (Breakwell 2004: 35). Als „vorgestellte Gemeinschaft“ (Anderson 2005), deren Angehörige sich erst über sozial konstruierte Gemeinsamkeiten (Eisenstadt/Giesen 1995) als zusammengehörig begreifen, lässt sie eine Vielzahl unterschiedlicher Interpretationen zu. Dabei dürfte die Erfahrbarkeit der EU zwischen den Ländern durchaus variieren. Vor dem Hintergrund der gemeinsamen Währung in der Eurozone oder des Verschwindens der Grenzkontrollen im Schengenraum kann für die entsprechenden Mitgliedsstaaten eine größere psychische Präsenz der EU angenommen werden, als es die von der Lebenswelt weitgehend abgekoppelte und abstrakte EU-Mitgliedschaft nahe legt. Folgt man dieser Perspektive, so ist die EU in jenen Ländern am stärksten erfahrbar, die nicht nur im Schengenraum, sondern auch in der Eurozone integriert sind. Am wenigsten erfahrbar ist sie in den Staaten, die lediglich EU-Mitgliedsstatus haben.

Das Konzept der Entitativity ergibt auch im Hinblick auf die Öffentlichkeitsforschung einen Mehrwert und ist als Erklärungsansatz nutzbar. Es kann vermutet werden, dass Gesellschaften, in denen die EU als relevante Größe im Alltag wahrgenommen wird, nicht nur verstärkte Aufmerksamkeit auf die europäische Politik richten, sondern auch eher in transnationale Diskurse eintreten als Gesellschaften, die sich ihrer Zugehörigkeit zur EU nur wenig bewusst sind. Entsprechend müssten sich vertikale und horizontale Europäisierungsprozesse in der Öffentlichkeit vor allem in Ländern vollziehen, die stark in die EU integriert sind und in denen deshalb auch die Erlebbarkeit des europäischen Zusammenhangs vergleichsweise stark ausgeprägt ist. Dies wäre konsistent mit dem Befund, dass die Öffentlichkeit bezogen auf Politikfelder, in denen die EU ausgeprägte Entscheidungskompetenzen hat, deutlicher europäisiert ist (Abschnitt 2).

In Bezug auf die Identitätsforschung dürften die Entitativity und der jeweilige situative Kontext der Selbstdefinition als EU-Bürger nicht nur das Ausmaß europäischer Identität in den Mitgliedsländern bestimmen. Zusätzlich sollte in dem Maße, in dem die Gruppenzugehörigkeit kontextabhängig konstruiert und aktualisiert werden kann, auch die inhaltliche Vorstellung von der EU situationsspezifisch variieren. Je nach individueller Erfahrbarkeit der EU und den Interessen, die sich aus dem jeweiligen Kontext ergeben, dürfte also die diffuse Kategorie EU inhaltlich unterschiedlich ausgedeutet werden. Ist etwa der gemeinsame Binnenmarkt von Bedeutung und durch den Wegfall von Handelsschranken erfahrbar, so dürfte die EU als Wirtschaftsgemeinschaft gesehen werden. Stehen stattdessen die Reisefreiheit und die Arbeitsmigration im Vordergrund des eigenen Erlebens, müsste die EU als multikulturelle Begegnungsgemeinschaft wahrgenommen werden. Die Erfahrung eines starken wirtschaftlichen Konkurrenzkampfes unter den Bedingungen von Billigarbeit und der Angst vor einem Dumping der Sozial- 
systeme lässt die EU im negativen Sinne als neoliberalen Wettbewerbsraum erscheinen. Nach Erkenntnissen des jüngsten Eurobarometers nennen EU-Bürger auf die Frage, was die EU für sie persönlich bedeute, vor allem die Reisefreiheit und die Möglichkeit, im EU-Ausland zu arbeiten und zu studieren (42\%), aber auch den Euro als gemeinsames Zahlungsmittel (33\%) und die Friedenssicherung auf dem Kontinent (25\%) (Eurobarometer 71). ${ }^{6}$ Wie die jeweiligen Ausdeutungen jeweils bewertet werden, ob eine Vorstellung von der EU als Wirtschaftsgemeinschaft also befürwortet wird oder die EU lieber als andere Art von Gemeinschaft gesehen würde, wird in den Umfragen des Eurobarometers nicht erhoben.

Weitere Hinweise zur inhaltlichen Ausdeutung der EU ergeben sich aus Inhaltsanalysen von Medienbeiträgen in diversen EU-Mitgliedsländern. Die entsprechenden Studien zeigen erhebliche nationale Unterschiede sowie enge Beziehungen zwischen der nationalen und der europäischen Identität (Angelucci von Bogdandy 2003, Díez Medrano 2003). So lässt sich etwa in Deutschland aufgrund der nationalsozialistischen Vergangenheit ein starkes europäisches Zugehörigkeitsgefühl finden, das mit einem Verständnis der EU als politische Gemeinschaft verbunden ist (Seidendorf 2007). In Großbritannien dagegen führt die historisch-kulturelle Abgrenzung vom Kontinent verbunden mit der Erinnerung an die frühere Weltmachtstellung des britischen Empire zu einem schwachen Integrationswillen und einer distanzierten, eher auf ökonomischer Zweckmäßigkeit basierenden Haltung zur EU (Díez Medrano 2003). Dass es in den unterschiedlichen Nationen auch Überschneidungen hinsichtlich der europäischen Identität geben kann, zeigen Risse und van de Steeg bezogen auf das Thema der HaiderDebatte. In ihrer Frame-Analyse identifizieren sie dabei zwei europaweit verwendete Identitäts-Frames, die Europa als Rechts- bzw. moralische Gemeinschaft rahmen (Risse/ van de Steeg 2003). Im Zeitverlauf lassen die Untersuchungen von Angelucci von Bogdandy (2003) und Seidendorf (2007) Annäherungen in den Ausdeutungen europäischer Identität zwischen den Ländern erkennen.

Mit dem Ausmaß des europäischen Zugehörigkeitsgefühls einerseits und der inhaltlichen Ausdeutung der EU andererseits sind zwei Dimensionen des Konstrukts europäische Identität angesprochen. Dass beide zusammenhängen, liegt auf der Hand. Konkrete Aussagen über die Verbindung zwischen Identitätsartikulationen und inhaltlichen Vorstellungen können jedoch anhand der Befunde der Eurobarometer-Umfragen nicht gemacht werden, da entsprechende Daten nur in unterschiedlichen Wellen erhoben wurden und in den wenigen einschlägigen Inhaltsanalysen ebenfalls jeweils nur eine der beiden Dimensionen erfasst wurde. Eine Verbindung zwischen Identitäts-Ausmaß und inhaltlicher Ausdeutung der EU ermöglichen aber zum Teil die qualitativen Interviews von Díez Medrano (2003). Die befragten Personen unterscheiden sich national deutlich hinsichtlich ihres europäischen Zugehörigkeitsgefühls und haben dabei ein je unterschiedliches Bild von Europa.

Insgesamt liefert die Identitätsforschung instruktive Befunde, die sich sowohl auf die Mikroebene individueller Einstellungen als auch auf die Identitätskonstruktion bestimmter soziokultureller Milieus beziehen. Anschlussmöglichkeiten zur Öffentlichkeitsforschung, die sich ebenfalls mit den Integrationsdefiziten in der EU befasst, erge-

6 Andere Inhalte, die von den Bevölkerungen mit der EU assoziiert werden, reihen sich folgendermaßen: Einfluss in der Weltpolitik (23\%), Demokratie (22\%), Geldverschwendung (20\%), kulturelle Vielfalt (19\%), Bürokratie (19\%), wirtschaftlicher Wachstum (16\%), Arbeitslosigkeit (13\%), Unsicherheiten in der Überwachung der EU-Grenzen (12\%), steigende Kriminalität (11\%), Verlust kultureller Identität (10\%), soziale Sicherheit (10\%) und andere Nennungen $(1 \%)$. 
ben sich vor allem in der Tradition der Bewegungsforschung, die die Konstruktion kollektiver Identitäten in öffentlichen Diskursen in den Blick nimmt. Die Fokussierung der Meinungs- und Einstellungsforschung auf die individuelle Identitätskonstruktion macht eine Verbindung mit der Öffentlichkeitsforschung dagegen schwierig. So bleibt unklar, inwiefern die individuellen Einstellungen im politischen Entscheidungssystem wahrnehmbar sind und damit analog zur öffentlichen Meinung die Europapolitik mit beeinflussen können. ${ }^{7}$ Um die Anschlussfähigkeit für solche makroperspektivischen Fragestellungen zu gewährleisten, ist es notwendig, das Konzept europäischer Identität auf der Ebene der kollektiven Meinungsbildung in den Mitgliedsstaaten zu verorten. Im Folgenden wird daher eine konzeptionelle Erweiterung der Identitätsforschung durch die Öffentlichkeitstheorie entwickelt.

\section{Identität als Resultat kollektiver Aushandlungsprozesse im öffentlichen Diskurs}

Indem sich die aktuelle Identitätsforschung stark an sozialpsychologischen Ansätzen orientiert, versteht sie die Zugehörigkeit zu einer Gemeinschaft unter einem konstruktivistischen Paradigma nicht mehr als angeboren und unveränderlich, sondern als sozial konstruiert (z. B. Breakwell 2004). Damit ist die europäische Identität je nach individuellem Erleben und sozialem Kontext veränderbar. Dieses anspruchsvolle theoretische Konzept wird in der empirischen Forschung zur europäischen Identität aber nicht zufriedenstellend eingelöst. Mit dem Fokus auf dem individuellen Zugehörigkeitsgefühl werden die gesellschaftlichen Interaktionen, in denen Identität kontextspezifisch herausgebildet und aktualisiert wird, nicht berücksichtigt. Individuen entwickeln aber Identitätsvorstellungen nicht isoliert voneinander (Mead 1973). Vielmehr wird in der kommunikativen Auseinandersetzung mit anderen eine kollektive Identität konstruiert. Wenngleich die Bewegungsforschung nicht die Konstruktion europäischer Identität, sondern Identitäten kleinerer soziokultureller Gruppen in den Mittelpunkt stellt, zeigen die einschlägigen Arbeiten zur kollektiven Identitätskonstruktion, dass im gesellschaftlichen Austausch über die Zugehörigkeit zu einer Gruppe ein „Wir-Gefühl“ entwickelt wird. Die kollektive Dimension von Identität betrifft also die Frage, welche Gruppenidentität eine Gesellschaft für sich aushandelt, welche Grenzen sie zieht und welche Charakteristika sie für sich als konstitutiv ansieht. Das hier vertretene Konzept kollektiver Identität geht insofern über die Aggregation von individuellen Identitäten hinaus, als dass diese nicht miteinander verrechnet, sondern unter Berücksichtigung unterschiedlicher Durchsetzungsstärken zu einem gemeinsamen Resultat verarbeitet werden. Gerade in Bezug auf die Herstellung kollektiv verbindlicher Entscheidungen in der Europapolitik ist die Frage zentral, welche kollektive Identität in einer Gesellschaft konstruiert wird. In welchem Maße begreift sich also die Gesellschaft eines Landes als mehr oder weniger der EU zugehörig und mit welcher Vorstellung von der EU ist diese Selbstverortung verbunden?

Um die kollektive Aushandlung europäischer Identität in angemessener Weise mit einbeziehen zu können, ist eine öffentlichkeitstheoretische Fundierung des IdentitätsKonzepts unerlässlich. Erst auf dieser Basis kann die Identitätskonstruktion im gesellschaftlichen Diskurs verortet werden. Wie die kollektiven Aushandlungsprozesse europäischer Identität aus öffentlichkeitstheoretischer Perspektive zu fassen sind, lässt sich am anschaulichsten anhand des „Forumsmodells“ (Gerhards/Neidhardt 1991) entwi-

7 Lediglich in Wahlen oder Referenden werden die individuellen Einstellungen direkt wirksam. In Bezug auf die Routineprozesse der politischen Entscheidungsfindung dürften diese lediglich vermittelt über öffentliche Diskurse als Einflussgrößen wirksam werden. 
ckeln. Neidhardt (1994: 7) beschreibt Öffentlichkeit als „ein offenes Kommunikationsforum für alle, die etwas sagen oder das, was andere sagen, hören wollen“. Darin werden Themen und Meinungen verschiedener Sprecher gesammelt und diskursiv verarbeitet. Als Output oder Resultat des Verarbeitungsprozesses entsteht das Kollektivprodukt öffentliche Meinung, das wiederum den Input für das politische System liefert (Gerhards/Neidhardt 1991). Im Idealmodell setzt sich im Diskurs das beste Argument durch und bereitet den Boden für belastbare Politikentscheidungen (Habermas 2008). Durch die öffentliche Prüfung der Sprecherbeiträge erfüllt das System Öffentlichkeit nicht nur eine Transparenz-, sondern auch eine Validierungsfunktion (Neidhardt 1994). Das Verarbeitungsresultat öffentliche Meinung ist somit mehr als die Summe der individuellen Sprecherbeiträge. Die aus der kritischen Verarbeitung resultierenden Sichtweisen lassen sich dabei zwar nicht notwendig als homogen, aber doch zumindest als reflektierten Dissens (Peters 2007) und als kollektiv anerkannt begreifen (Eilders 2008). Die Öffentlichkeitsforschung nimmt an, dass moderne Öffentlichkeiten von Medien getragen werden und auf dieser Ebene institutionalisiert sind. Medien werden in diesem Sinne als wesentliche Foren der Identitätskonstruktion begriffen. ${ }^{8}$ Im Mediendiskurs wird die öffentliche Meinung als kollektiver Output des Verarbeitungssystems Öffentlichkeit sichtbar. Obwohl die Medienöffentlichkeit den hohen Ansprüchen, die das Idealmodell an Diskursivität und Gleichheit anlegt, nicht immer genügt, ist dort ablesbar, welche Themen und Meinungen in der öffentlichen Diskussion als relevant und akzeptiert gelten. Der Mediendiskurs dient daher der Politik als einfach zugängliche Orientierungsmarke.

Auch die europäische Identität einer Gesellschaft wird in der Medienöffentlichkeit kollektiv ausgehandelt. Sie ist Teil des gesellschaftlichen Selbstverständnisses und eine soziale, aus der Verarbeitung individueller Sichtweisen resultierende Konstruktion (Angelucci von Bogdandy 2003). Dabei tauschen verschiedene Sprechergruppen vor einem Publikum ihre Zugehörigkeitsartikulationen sowie ihre identitätsrelevanten Deutungen zur EU aus. Die Sprecher weisen der Zugehörigkeit zur EU im Verhältnis zu anderen Identitäten in je verschiedenem Maße Relevanz zu und beziehen sich positiv, gleichgültig oder skeptisch auf sie. Unterschiedliche Positionen können sich in diesem Prozess einander annähern, müssen aber keinesfalls zu einer homogenen Sichtweise verschmelzen. Vielmehr können auch dauerhaft miteinander konkurrierende Konstruktionen nebeneinander bestehen bleiben. Das Resultat sind kollektiv reflektierte und weitgehend akzeptierte Identitätsvorstellungen. Die kollektive Identität einer Gesellschaft nimmt in dieser Perspektive erst im öffentlichen Diskurs Gestalt an. Sie ist über die Medien gut wahrnehmbar, so dass sie als Orientierung für europapolitische Entscheidungen dienen kann.

Öffentlich ausgehandelt werden sowohl die kontextspezifische Stärke der kollektiven europäischen Identität als auch das Vorstellungsbild, das mit der EU verbunden wird und auf das sich das Zugehörigkeitsgefühl richtet. Die inhaltliche Ausdeutung der EU kann unterschiedlich bewertet werden, was sich wiederum auf das Zugehörigkeitsgefühl auswirken kann. Dabei dürfte etwa ein ausgeprägtes kollektives Zugehörigkeitsgefühl zur EU mit einem Bild der EU als Solidar- oder als Friedensgemeinschaft und einer positiven Bewertung dieses Bildes zusammenhängen, eine schwach empfundene europäische Zugehörigkeit dagegen zum Beispiel mit der negativ bewerteten Vorstellung eines neoliberalen Wettbewerbsraums oder der Furcht, Besonderheiten der nationalen

8 Indem Medien als Ort der Identitätskonstruktion gekennzeichnet werden, spielen Wirkungsüberlegungen hier keine Rolle. Zur Kritik an entsprechenden Kausalzusammenhängen vgl. z. B. Hepp (2006) und Schlesinger (1987). 
Kultur durch die Integration zu verlieren. Die Art und Weise, wie eine mehr oder weniger ausgeprägte europäische Identität inhaltlich bestimmt und begründet wird, lässt sich als Framing beschreiben. Mit diesem Begriff wird ein in der Öffentlichkeitsforschung sowie in der Bewegungsforschung üblicher Zugang zum Mediendiskurs auf die Identitätsforschung übertragen. Dabei lassen sich die identitätsbezogenen Deutungen der EU als Identitätsframes untersuchen. Versteht man einen Frame als spezifische Rahmung eines Themas oder Sachverhalts, die über Selektion und Salienzzuweisung (Matthes 2007) einige Aspekte hervorhebt und dadurch eine bestimmte Perspektive auf den Gegenstand vorgibt, ohne schon explizit zu bewerten (Eilders 2004), legen Identitätsframes die Interpretation der EU als spezifische Art von Gemeinschaft fest, ohne dass damit auch das Ausmaß an Zugehörigkeit determiniert wäre.

Im Diskurs kristallisieren sich aus der Vielfalt existenter Deutungsangebote ${ }^{9}$ einige Identitätsinhalte heraus, die für die soziale Kategorie EU maßgebend werden. Sie sind mit politischen Implikationen verbunden: Über Identitätsframes werden Grenzen, Werte und Ziele einer Gemeinschaft sowie Erwartungen und Forderungen an die gemeinsame Politik (Lepsius 2004) begründet. Sie beinhalten z. B. narrative Elemente, um die Kontinuität einer Gemeinschaft zu beschreiben (Anderson 2005), und Codes, mit Hilfe derer sich eine Gruppe von anderen unterscheiden lässt (Eisenstadt/Giesen 1995). Aus unterschiedlichen Identitätsframes können entsprechend eng bzw. weit gefasste Zugehörigkeiten und stabile bzw. variable Grenzen resultieren. Insofern ist die Identitätsvorstellung von der EU beispielsweise als kulturelle oder primär wirtschaftliche $\mathrm{Ge}-$ meinschaft auch für die Finalität der Unionsgrenzen von hoher Relevanz.

Dass sich in den europäischen Ländern die gleichen Identitätsframes herausbilden, ist unwahrscheinlich. Dies würde zumindest einen intensiven Kommunikationszusammenhang voraussetzen, der von der Öffentlichkeitsforschung angezweifelt wird (siehe Abschnitt 2). Die Reichweite der Medien als primäre Arenen der Identitätskonstruktion beschränkt sich vor allem auf den nationalen Raum, und auch soziale und politische Bedürfnisse werden zuerst im nationalen Diskurs transparent. Auch die Heterogenität der etablierten nationalen Identitäten in Europa, die eine europäische Identität jeweils unterschiedlich beeinflussen, sprechen gegen die Annahme übereinstimmender Identitätsvorstellungen innerhalb der EU. Wahrscheinlicher ist eine Vielzahl kollektiver europäischer Identitäten, die jeweils national unterschiedliche Akzentuierungen beinhalten (Marcussen et al. 1999, Risse 2001). Dabei ist auch jede national gefärbte Identitätskonstruktion immer nur vorläufig: Wenn sich die politischen, sozialen und wirtschaftlichen Bedingungen wandeln, neue Länder beitreten oder sich auf andere Weise ein Anpassungsdruck oder Konfliktstoff ergibt, kann neuer Bedarf zur gesellschaftlichen Selbstverständigung entstehen (Risse 2001). Konflikte sind insofern nicht nur für die Entstehung europäischer Öffentlichkeit konstruktiv, sondern stellen auch Anlässe für die diskursive Auseinandersetzung mit der europäischen Identität dar. Besonders deutlich zeigte sich das 2005 in der französischen Öffentlichkeit, als der Diskurs über die EU im Vorfeld des Verfassungsreferendums allgegenwärtig war.

9 Grob lässt sich unterscheiden, ob die EU vor allem als politische, wirtschaftliche oder kulturelle Gemeinschaft betrachtet wird oder auch nur als geografische Einheit. Jede dieser vier allgemeinen Deutungen lässt sich in konkretere Frames unterteilen. So kann von der Idee der Kulturgemeinschaft eine Werte-, eine Religions- oder eine historische Gemeinschaft abgeleitet werden. 


\section{Fazit}

Ausgangspunkt der Überlegungen zum Integrationsdefizit der EU war das mangelnde Zugehörigkeitsgefühl der europäischen Bevölkerungen. Während sich die Forschung bislang auf das Öffentlichkeitsdefizit der EU konzentriert und umfassende theoretische Analysen und empirische Befunde vorgelegt hat, besteht in Bezug auf die europäische Identität noch erheblicher Nachholbedarf. In theoretischer Perspektive betrifft das die Erweiterung des Identitätsverständnisses vom individuellen Zugehörigkeitsgefühl auf das kollektive Selbstverständnis einer Gesellschaft. In empirischer Hinsicht folgt daraus eine Ergänzung der bislang vorherrschenden empirischen Erfassung von Identität auf der Individualebene mittels Umfragen, indem nun - analog zum Vorgehen in Öffentlichkeits- und Bewegungsforschung - gesellschaftliche Diskurse in den Forschungsfokus rücken. Das sozialpsychologisch inspirierte Verständnis von Identität als soziale Konstruktion ist zwar vielversprechend, die Konstruktion wird jedoch nicht konsequent in der Gesellschaft verortet und entsprechend auch nicht in der sozialen Interaktion erfasst. Vielmehr beschränkt sich ein Großteil der Forschung auf die individuellen Zugehörigkeitsgefühle und verliert dabei den gesellschaftlichen Kontext aus dem Blick. Durch die Konzentration auf die Individualebene hat sich die Identitätsforschung zwar nicht mit dem in der Öffentlichkeitsforschung problematischen Elite-Bias auseinanderzusetzen, allerdings wird damit die Chance vertan, die EU-relevanten Entscheidungen im politischen System mit der europäischen Identität systematisch in Beziehung setzen zu können. Schließlich sind die individuellen Einstellungen der Bürger im Gegensatz zu den Meinungen, die im öffentlichen Diskurs zum Ausdruck kommen, für die Entscheidungsebene nur sehr eingeschränkt wahrnehmbar.

Dieser Beitrag hat die bisher getrennt verlaufenden Forschungsstränge zur europäischen Öffentlichkeit und Identität miteinander verbunden, indem er eine Erweiterung der Identitätsforschung durch öffentlichkeitstheoretische Konzepte vorgeschlagen hat. Diese setzt bei einer Zusammenführung des Konzepts kollektiver Identität mit dem Framing-Ansatz an, die sich bereits in den Forschungen zu sozialen Bewegungen als fruchtbar erwiesen hat. Europäische Identität wird als kollektiv hergestellte und sich dynamisch entwickelnde Konstruktion verstanden, die aus dem permanent ablaufenden diskursiven Selbstverständigungsprozess einer Gesellschaft resultiert. Die Identitätskonstruktionen werden als Teil der öffentlichen Meinung konzipiert und damit auf der Makroebene angesiedelt. Dabei gehen nicht alle individuellen Konstruktionen mit gleichem Gewicht in das kollektive Selbstverständnis ein, sondern es setzen sich bestimmte Sichtweisen und Deutungen durch, während andere nicht auf Akzeptanz stoßen. Ebenso wie die öffentliche Meinung ist die kollektive Identität damit mehr als die Summe ihrer Teile. Die diskursive Auseinandersetzung über die europäische Identität findet in den überwiegend national geprägten Öffentlichkeiten der EU-Mitgliedsstaaten statt, die im Wesentlichen über die nationalen Medien zugänglich sind. Mit dem Verständnis von Identität als diskursive Konstruktion eröffnet sich daher auch ein erweiterter methodischer Zugang zur europäischen Identität. Inhaltsanalysen der nationalen Mediendiskurse rücken so in den Mittelpunkt der Identitätsforschung.

Die europäische Identität als kollektive Konstruktion wurde als zwei-dimensionales Konstrukt begriffen. Die eine Dimension betrifft die gefühlte Zugehörigkeit zu einer Gemeinschaft („wer sind wir - z. B. Deutsche und/oder EU-Bürger?“), die andere die inhaltliche Ausdeutung oder auch das Framing dieser Gemeinschaft („was sind wir z. B. eine Werte- oder eine Wirtschaftsgemeinschaft?“) mitsamt deren Bewertung („befürworten wir die EU als Werte- bzw. Wirtschaftsgemeinschaft?"). Die Identitätsframes begründen das Zugehörigkeitsgefühl zur EU, indem sie zum Beispiel allgemein akzep- 
tierte Werte ansprechen, wie etwa Frieden oder Rechtsstaatlichkeit. Durch das jeweilige Framing wird eine bestimmte Bewertung nahegelegt, aber nicht determiniert. Auch die gefühlte Zugehörigkeit hängt mit inhaltlichen Ausdeutungen und deren Bewertung zusammen, ohne von diesen aber festgelegt zu sein. Denkbar ist etwa, dass die EU vor allem als Solidargemeinschaft gesehen wird, die Gesellschaft eines Landes diese Deutung jedoch negativ bewertet und sich entsprechend nicht als europäisch definiert. Ebenso kann eine Gesellschaft die EU als Friedensgemeinschaft positiv bewerten und trotzdem nicht zu starken Identitätsartikulationen motiviert werden. Der Framing-Ansatz ist vor allem in der stark inhaltsanalytisch arbeitenden Öffentlichkeitsforschung verbreitet. Die Anwendung in der Identitätsforschung zeigt, dass die öffentlichkeitstheoretische Fundierung der Identitätsforschung nicht nur auf der Ebene von Basiskonzepten, sondern auch in Bezug auf konkrete Forschungsansätze und methodische Zugänge ertragreich ist.

Auch die Öffentlichkeitsforschung kann durch die Integration der beiden Forschungsstränge profitieren. Zum einen verweist das Verständnis von Identität als kollektives Aushandlungsresultat auf neue Fragestellungen für die Analyse öffentlicher Diskurse. Bei der Untersuchung von synchroner Berichterstattung in den einzelnen EULändern geraten neben den EU-relevanten Politikfeldern, die bislang die Öffentlichkeitsforschung dominiert haben, nun vor allem identitätsrelevante Inhalte in den Blick. Eine europäische Öffentlichkeit konstituiert sich in dieser Perspektive nicht nur durch parallele Themen und Frames, unter denen diese Themen verhandelt werden. Vielmehr nimmt sie auch Gestalt an, indem entweder die europäische Identität übereinstimmend artikuliert oder aber kontrovers diskutiert wird. Zum anderen bietet das innerhalb der Identitätsforschung entwickelte Konzept der Entitativity die Möglichkeit, konkrete Erwartungen in Bezug auf die Europäisierung der Öffentlichkeit zu formulieren. Der Integrationsgrad eines Landes dürfte sich in der Erlebbarkeit der EU im Alltag und schließlich auf die Identitätskonstruktionen im öffentlichen Diskurs niederschlagen. Aufgrund der in den EU-Mitgliedsländern unterschiedlich stark ausgeprägten Entitativity lässt sich vermuten, dass die europäische Identität in den nationalen Diskursen unterschiedlich konstruiert wird. So dürften die Reisefreiheit im Schengenraum oder etwa die gemeinsame Währung in der Eurozone zu einer verstärkten europäischen Identitätskonstruktion innerhalb der nationalen Diskurse beitragen und die Bezüge auf die EU-Ebene sowie den transnationalen Diskurs befördern. Folglich dürften sowohl die vertikalen als auch die horizontalen EU-Bezüge im öffentlichen Diskurs wesentlich von der Entitativity abhängen, die ihrerseits auch über die supranationalen Bezüge und den transnationalen Diskurs erhöht wird. In welchem Maße in den nationalen Mediendiskursen eine europäische Identität konstruiert wird und welche Gemeinsamkeiten zwischen den EU-Staaten dabei sichtbar werden, ist eine empirische Frage, die zunächst offen bleiben muss. Die hier ausgeführte theoretische Neukonzeption der Identitätsforschung sollte dabei eine instruktive Anleitung für zukünftige empirische Forschungsaktivitäten sein.

\section{Literatur}

Anderson, Benedict (2005): Die Erfindung der Nation. Zur Karriere eines folgenreichen Konzepts. 2. Auflage. Frankfurt am Main/New York: Campus.

Angelucci von Bogdandy, Orietta (2003): Zur Ökologie einer europäischen Identität. Soziale Repräsentationen von Europa und dem Europäer-Sein in Deutschland und Italien. Baden-Baden: Nomos.

Berkel, Barbara (2006): Konflikt als Motor europäischer Öffentlichkeit. Eine Inhaltsanalyse von Tageszeitungen in Deutschland, Frankreich, Großbritannien und Österreich. Wiesbaden: VS. 
Brantner, Cornelia (2009): Medien und EU: Europäisierung der österreichischen Öffentlichkeit? Eine Inhaltsanalyse des öffentlichen Diskurses zur Osterweiterung der Europäischen Union und zum EU-Beitritt der Tschechischen Republik. Saarbrücken: Südwestdeutscher Verlag für Hochschulschriften.

Breakwell, Glynis M. (2004): Identity Change in the Context of the Growing Influence of European Union Institutions. In: Herrmann, Richard K./Risse, Thomas/Brewer, Marilynn B. (Hrsg.), Transnational Identities. Becoming European in the EU. Oxford: Rowman \& Littlefield, S. 25-39.

Brewer, Marilynn B. (2009): Social Identity and Citizenship in a Pluralistic Society. In: Borgida, Eugene/Federico, Christopher M./Sullivan, John L. (Hrsg.), The Political Psychology of Democratic Citizenship. Oxford: Oxford University Press, S. 153-175.

Brewer, Marilynn B. (1991): The Social Self. On Being the Same and Different at the Same Times. In: Personality and Social Psychology Bulletin, Vol. 17(5), S. 475-482.

Brüggemann, Michael/Hepp, Andreas/Kleinen-von Königslöw, Katharina/Wessler, Hartmut (2009): Transnationale Öffentlichkeit in Europa. Forschungsstand und Perspektiven. In: Publizistik 3/2009, S. 391-414.

Campbell, Donald T. (1958): Common Fate, Similarity, and Other Indices of the Status Aggregates of Persons as Social Entities. In: Behavioral Science, Vol. 3, S. 14-25.

Castano, Emanuele (2004): European Identity: A Social-Psychological Perspective. In: Herrmann, Richard K./Risse, Thomas/Brewer, Marilynn B. (Hrsg.), Transnational Identities. Becoming European in the EU. Oxford: Rowman \& Littlefield, S. 40-58.

Castano, Emanuele/Yzerbyt, Vincent/Bourguignon, David (2003): We Are One and I Like It: The Impact of Group Entitativity on Ingroup Identification. In: European Journal of Social Psychology, Vol. 33, S. 735-754.

de Vreese, Claes/Boomgaarden, Hajo G. (2005): Projecting EU Referendums. Fear of Immigration and Support for European Integration. In: European Union Politics, Vol. 6(1), S. 59-82.

Díez Medrano, Juan (2003): Framing Europe. Attitudes to European Integration in Germany, Spain, and the United Kingdom. Princeton Mass/Oxford: Princeton University Press.

Downey, John/König, Thomas (2006): Is There a European Public Sphere? The Berlusconi-Schulz Case. In: European Journal of Communication, Vol. 21(2), S. 165-187.

Eder, Klaus (2000): Kulturelle Identität zwischen Tradition und Utopie. Soziale Bewegungen als Ort gesellschaftlicher Lernprozesse. Frankfurt am Main/New York: Campus.

Eder, Klaus/Kantner, Cathleen (2000): Transnationale Resonanzstrukturen in Europa. Eine Kritik der Rede vom Öffentlichkeitsdefizit. In: Bach, Maurizio (Hrsg.), Die Europäisierung nationaler Gesellschaften. Kölner Zeitschrift für Soziologie und Sozialpsychologie. Sonderheft 40. Opladen: Westdeutscher Verlag, S. 306-332.

Eichenberg, Richard/Dalton, Russell J. (2007): Post-Maastricht-Blues: The Transformation of Citizen Support for European Integration, 1973-2004. In: Acta Politica, Vol. 42(2-3), S. 128-152.

Eilders, Christiane (2008): Massenmedien als Produzenten öffentlicher Meinungen - Pressekommentare als Manifestation der politischen Akteursrolle. In: Pfetsch, Barbara/Adam, Silke (Hrsg.), Massenmedien als politische Akteure. Konzepte und Analysen. Wiesbaden: VS, S. 27-51.

Eilders, Christiane (2004): Von Links nach Rechts - Deutung und Meinung in Pressekommentaren. In: Eilders, Christiane/Neidhardt, Friedhelm/Pfetsch, Barbara (Hrsg.), Die Stimme der Medien. Pressekommentare und politische Öffentlichkeit in der Bundesrepublik. Wiesbaden: VS, S. 129-166.

Eilders, Christiane/Lüter, Albrecht (2000): Germany at War: Competing Framing Strategies in German Public Discourse. In: European Journal of Communication, Vol. 15(3), S. 415-428.

Eilders, Christiane/Voltmer, Katrin (2004): Zwischen Marginalisierung und Konsens: Europäische Öffentlichkeit in Deutschland. In: Eilders, Christiane/Neidhardt, Friedhelm/Pfetsch, Barbara (Hrsg.), Die Stimme der Medien. Pressekommentare und politische Öffentlichkeit in der Bundesrepublik. Wiesbaden: VS, S. 358-383.

Eilders, Christiane/Voltmer, Katrin (2003): Zwischen Deutschland und Europa. Eine empirische Untersuchung zum Grad von Europäisierung und Europaunterstützung der meinungsführen- 
den deutschen Tageszeitungen. In: Medien \& Kommunikationswissenschaft, Vol. 51(2), S. 250-270.

Eisenstadt, Shmuel N./Giesen, Bernhard (1995): The Construction of Collective Identity. In: European Journal of Sociology, Vol. 36, S. 72-102.

Engelmann, Ines (2009): Der mediale Diskurs über die EU-Osterweiterung. Europäisierung der deutschen Medienöffentlichkeit am Beispiel zweier Qualitätszeitungen. In: Medien \& Kommunikationswissenschaft, Vol. 57 (1), S. 39-55.

European Commission (2009): Eurobarometer 72. Public Opinion in the European Union. First Results. Fieldwork: October-November 2009. http://ec.europa.eu/public_opinion/archives/ eb/eb72/eb72_first_en.pdf [02.05.2010].

European Commission (2008): Eurobarometer 68. Public Opinion in the European Union. Fieldwork: September-November 2007. http://ec.europa.eu/public_opinion/archives/eb/eb68/ eb_68_en.pdf [02.05.2010].

European Commission (2006): Eurobarometer 64. Die öffentliche Meinung in der europäischen Union. Befragung: Oktober-November 2005. http://ec.europa.eu/public_opinion/archives/ eb/eb64/eb64_de.pdf [02.05.2010].

Gamson, William A. (1992): The Social Psychology of Collective Action. In: Morris, Aldon D./ Mueller, Carol McClough (Hrsg.), Frontiers in Social Movement Theory. New Haven, CT: Yale University, S. 53-76.

Gamson, William A./Fireman, Bruce/Rytina, Steven (1982): Encounters with Unjust Authority, Homewood, Illinois: Dorsey Press.

Gamson, William A./Meyer, David S. (1996): Framing Political Opportunity. In: McAdam, Doug/ McCarthy, John/Zald, Mayer N. (Hrsg.), Comparative Perspectives on Social Movements. Political Opportunities, Mobilizing Structures, and Cultural Framings. Cambridge: Cambridge University Press, S. 275-290.

Gerhards, Jürgen (2000): Europäisierung von Ökonomie und Politik und die Trägheit der Entstehung von Öffentlichkeit. In: Bach, Maurizio (Hrsg.), Die Europäisierung nationaler Gesellschaften. Kölner Zeitschrift für Soziologie und Sozialpsychologie. Sonderheft 40. Opladen: Westdeutscher Verlag, S. 277-305.

Gerhards, Jürgen/Neidhardt, Friedhelm (1991): Strukturen und Funktionen moderner Öffentlichkeit. Fragestellungen und Ansätze. In: Müller-Doohm, Stefan/Neumann-Braun, Klaus (Hrsg.), Öffentlichkeit, Kultur, Massenkommunikation. Oldenburg: Universitätsverlag, S. 31-90.

Gerhards, Jürgen/Rucht, Dieter (1992): Mesomobilization: Organizing and Framing in Two Protest Campaigns in West Germany. In: American Journal of Sociology, Vol. 98, S. 555-595.

Grimm, Dieter (1995): Braucht Europa eine Verfassung? München: C. H. Beck.

Grundmann, Reiner/Smith, Dennis/Wright, Sue (2000): National Elites and Transnational Discourses in the Balkan War. A Comparison between the French, German and British Establishment Press. In: European Journal of Communication, Vol. 15(3), S. 299-320.

Habermas, Jürgen (2008): Ach, Europa. Kleine Politische Schriften XI. Frankfurt am Main: Suhrkamp.

Habermas, Jürgen (2004): Der gespaltene Westen. Kleine Politische Schriften X. Frankfurt am Main: Suhrkamp.

Hall, Stuart (1994): Rassismus und kulturelle Identität. Ausgewählte Schriften 2. Hamburg: Argument.

Haunss, Sebastian (2004): Identität in Bewegung. Prozesse kollektiver Identität bei den Autonomen und in der Schwulenbewegung. Wiesbaden: VS.

Hepp, Andreas (2006): Transkulturelle Kommunikation. Konstanz: UVK.

Hepp, Andreas/Wessler, Hartmut (2009): Politische Diskurskulturen. Überlegungen zur empirischen Erklärung segmentierter europäischer Öffentlichkeit. In: Medien \& Kommunikationswissenschaft, Vol. 57(2), S. 174-197.

Herrmann, Richard K./Risse, Thomas/Brewer, Marilynn B. (Hrsg.) (2004): Transnational Identities. Becoming European in the EU. Oxford: Rowman \& Littlefield.

Imhof, Kurt (2003): Öffentlichkeit und Identität. In: Winter, Carsten/Thomas, Tanja/Hepp, Andreas (Hrsg.), Medienidentitäten. Identität im Kontext von Globalisierung und Medienkultur. Köln: Halem, S. 71-93. 
Kalantzi, Martha (2004): Europa in der Tagespresse Deutschlands und Griechenlands. In: Hagen, Lutz M. (Hrsg.), Europäische Union und mediale Öffentlichkeit. Theoretische Perspektiven und empirische Befunde zur Rolle der Medien im europäischen Einigungsprozess. Köln: Halem, S. 178-194.

Kantner, Cathleen (2004): Kein modernes Babel. Kommunikative Voraussetzungen europäischer Öffentlichkeit. Wiesbaden: VS.

Kielmansegg, Peter Graf (1996): Integration und Demokratie. In: Jachtenfuchs, Markus/KohlerKoch, Beate (Hrsg.), Europäische Integration. Opladen: Leske+Budrich, S. 47-83.

Koopmans, Ruud (2004): Integrated Report: Cross-National, Cross-Issue, Cross-Time. Project: The Transformation of Political Mobilisation and Communication in European Public Spheres. Integrated Report of WP 2. http://europub.wzb.eu/Data/reports/WP2/D2-4 \%20WP2\%20 Integrated\%20Report.pdf [02.05.2010].

Lepsius, M. Rainer (2004): Prozesse der europäischen Identitätsstiftung. In: Aus Politik und Zeitgeschichte 38, S. 3-13.

Lucht, Jens/Tréfas, David (2006): Hat Europa eine Identität? Eine zeitreihenbasierte Untersuchung der öffentlichen europäischen Kommunikation von 1951-2005. Fög Discussion Paper DI-2006-0001. Zürich: Fög - Forschungsbereich Öffentlichkeit und Gesellschaft. http:// www.foeg.unizh.ch/staging/userfiles/file/Deutsch/f\%C3\%B6g\%20discussion\%20papers/ DI-2006-0001_hat_europa_eine_identitaet.pdf [02.05.2010].

Marcussen, Martin/Risse, Thomas/Engelmann-Martin, Daniela/Knopf, Hans Joachim/Roscher, Klaus (1999): Constructing Europe? The Evolution of French, British and German NationState Identities. In: Journal of European Public Policy, Vol. 6(4), S. 614-633.

Matthes, Jörg (2007): Framing-Effekte. Zum Einfluss der Politikberichterstattung auf die Einstellungen der Rezipienten. München: Reinhard Fischer.

Mead, George H. (1973): Geist, Identität und Gesellschaft. Aus der Sicht des Sozialbehaviorismus. Mit einer Einleitung herausgegeben von Charles W. Morris. Frankfurt am Main: Suhrkamp.

Morley, David/Robins, Kevin (2002): Spaces of Identity. Global Media, Electronic Landscapes and Cultural Boundaries. 4. Auflage. London/New York: Routledge.

Neidhardt, Friedhelm (2006): Europäische Öffentlichkeit als Prozess. Anmerkungen zum Forschungsstand. In: Langenbucher, Wolfgang R./Latzer, Michael (Hrsg.), Europäische Öffentlichkeit und medialer Wandel. Eine transdisziplinäre Perspektive. Wiesbaden: VS, S. 46-61.

Neidhardt, Friedhelm (1994): Öffentlichkeit, öffentliche Meinung, soziale Bewegung. In: Ders. (Hrsg.), Öffentlichkeit, öffentliche Meinung, soziale Bewegung. Kölner Zeitschrift für Soziologie und Sozialpsychologie. Sonderheft 34. Opladen: Westdeutscher Verlag, S. 7-41.

Peter, Jochen/de Vreese, Claes (2004): In Search of Europe: A Cross-National Comparative Study of the European Union in National Television News. In: Havard Journal of Press/Politics, Vol. 9(4), S. 3-24.

Peters, Bernhard (2007): Der Sinn von Öffentlichkeit. Frankfurt am Main: Suhrkamp.Risse, Thomas (2004): European Institutions and Identity Change: What Have We Learned? In: Herrmann, Richard K./Risse, Thomas/Brewer, Marilynn B. (Hrsg.), Transnational Identities. Becoming European in the EU. Oxford: Rowman \& Littlefield, S. 247-271.

Risse, Thomas (2001): A European Identity? Europeanization and the Evolution of Nation-State Identities. In: Green Cowles, Maria/Caporaso, James/Risse, Thomas (Hrsg.), Transforming Europe. Europeanization and Domestic Change. Ithaca/London: Cornell University Press, S. 198-216.

Risse, Thomas/van de Steeg, Marianne (2003): An Emerging European Public Sphere? Empirical Evidence and Theoretical Clarifications. Discussion Paper. Europeanisation of Public Spheres, Political Mobilisation, Public Communication and the European Union, Science Center Berlin, 20.-22. Juni 2003. http://www.polsoz.fu-berlin.de/polwiss/forschung/international/atasp/pu blikationen/4_artikel_papiere/21/030624_europeanpublicsphere.pdf [02.05.2010].

Saurwein, Florian/Brantner, Cornelia/Dietrich, Astrid (2006): Europäisierung der österreichischen Öffentlichkeit. Mediale Aufmerksamkeit für EU-Politik und der veröffentlichte Diskurs über die EU-Erweiterung. Forschungsbericht. Wien: Institut für Publizistik und Kommunikationswissenschaft. 
Schlesinger, Philip (1987): On National Identity. In: Social Science Information, Vol. 26(2), S. 219-264.

Schmale, Wolfgang (2008): Geschichte und Zukunft der Europäischen Identität. Stuttgart: Kohlhammer.

Seidendorf, Stefan (2007): Europäisierung nationaler Identitätsdiskurse? Ein Vergleich französischer und deutscher Printmedien. Baden-Baden: Nomos.

Sievert, Holger (1998): Europäischer Journalismus. Theorie und Empirie aktueller Medienkommunikation in der Europäischen Union. Opladen/Wiesbaden: Westdeutscher Verlag.

Snow, David A./Benford, Robert D. (1988): Ideology, Frame Resonance, and Participant Mobilization. In: Klandermans, Bert/Kriesi, Hanspeter/Tarrow, Sidney (Hrsg.), International Social Movement Research, 1. Greenwich/Connecticut: JAI Press,, S. 197-217.

Snow, David A./Benford, Robert D. (1992): Master Frames and Cycles of Protest. In: Morris, Aldon D./Mueller, Carol McClough (Hrsg.), Frontiers in Social Movement Theory. New Haven, CT: Yale University, S. 133-155.

Snow, David A./Rochford, E. Burke/Worden, Steven K./Benford, Robert D. (1986): Frame Alignment Processes, Micromobilization and Movement Participation. In: American Sociological Revue, Vol. 51(4), S. 456-581.

van de Steeg, Marianne (2003): Bedingungen für die Entstehung von Öffentlichkeit in der EU. In: Klein, Ansgar/Koopmans, Ruud/Trenz, Hans-Jörg (Hrsg.), Bürgerschaft, Öffentlichkeit und Demokratie in Europa. Opladen: Leske+Budrich, S. 169-190.

Taylor, Verta/Whittier, Nancy (1992): Collective Identity in Social Movement Communities: Lesbian Feminist Mobilization. In: Morris, Aldon D./Mueller, Carol McClough (Hrsg.), Frontiers in Social Movement Theory. New Haven, CT: Yale University, S. 104-129.

Trenz, Hans-Jörg (2004): Media Coverage on European Governance. Exploring the European Public Sphere in National Quality Newspapers. In: European Journal of Communication, Vol. 19(3), S. 291-319.

Trenz, Hans-Jörg (2000): Korruption und politischer Skandal in der EU. Auf dem Weg zu einer europäischen politischen Öffentlichkeit? In: Bach, Maurizio (Hrsg.), Die Europäisierung nationaler Gesellschaften. Kölner Zeitschrift für Soziologie und Sozialpsychologie. Sonderheft 40. Opladen: Westdeutscher Verlag, S. 332-359.

Wessler, Hartmut/Peters, Bernhard/Brüggemann, Michael/Kleinen-von Königslöw, Katharina; Sifft, Stefanie (2008): Transnationalization of Public Spheres. Basingstoke: Palgrave Macmillan.

Wimmer, Jeffrey (2003): Identität der Gegenöffentlichkeit - Proteste gegen die Liberalisierung des Welthandels. In: Winter, Carsten/Thomas, Tanja/Hepp, Andreas (Hrsg.), Medienidentitäten. Identität im Kontext von Globalisierung und Medienkultur. Köln: Halem, S. 362-375.

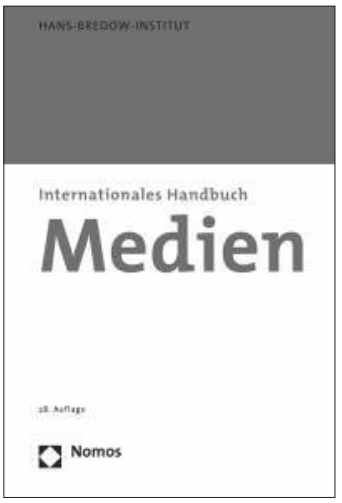

\section{Internationales Handbuch Medien}

Vom Hans-Bredow-Institut

28. Auflage 2009, 1.308 S., geb., 128,- $€$, ISBN 978-3-8329-3423-1

"Wie schon frühere Ausgaben, ist das Buch ohne Konkurrenz - nicht nur im deutschsprachigen Raum." Harald Kuhl, Radio-Kurier 9/o9 